\section{Preface \\ Message from the Guest Editors-In-Chief}

\author{
Hajime Nagahara, ${ }^{\dagger 1}$ Ryusuke Sagawa, ${ }^{\dagger 2}$ \\ PASCAL VASSEUR ${ }^{\dagger 3}$ and SHrEe K. NAYAR ${ }^{\dagger 4}$
}

issue and all the reviewers who contribute to improve the quality of this issue.

\section{Guest Editors in Chief:}

Hajime Nagahara Kyushu University

Ryusuke Sagawa Advanced Industrial Science and Technology

Pascal Vasseur

University of Picardie

Shree K. Nayar Columbia University

We are grad to have the special issue of Omnidirectional Vision, Camera Networks and Non-classical Cameras. This special issue is conjunction with the ninth workshop on Omnidirectional Vision, Camera Networks and Non-classical Cameras (OMNIVIS), held in Kyoto, Japan, on October 4th, 2009. The OMNIVIS Workshop continues a series of workshops started in 2000. The aim of the workshop is to bring together researchers interested in camera networks and omnidirectional vision including novel imaging and projection systems and catadioptric systems.

We widely accepted not only the papers appeared in OMNIVIS2009 but also the outside of the papers related to the topic. As the result of strict review process, we have decided that two high quality papers are appeared in this issue. The paper: "Krill-eye: Superposition Compound Eye for Wide-Angle Imaging via GRIN Lenses" by Sinsaku Hiura, Ankit Mohan and Ramesh Raskar proposes a new concept for wide-angle imaging. The paper: "Video Completion for Generating Omnidirectional Video without Invisible Areas" by Norihiko Kawai, Kotaro Machikita, Tomokazu Sato, Naokazu Yokoya proposes a new processing pipeline to get a full field of view for computer graphics application.

We hope that both papers would give impact to robotics, computer vision and graphics communities.

We would like to thank again all the authors who submitted to this special

$\dagger 1$ Kyushu University

$\dagger 2$ Advanced Industrial Science and Technology

$\uparrow 4$ Columbia University 